The emergence of antimicrobial resistance (AMR) among sexually transmitted infections (STI) is a cause for global concern, and is epitomised by the fact we are now running out of treatment options for gonorrhoea. The role of AMR surveillance is now more important than ever. Ideally, AMR surveillance should be fast, easy, inexpensive, accessible, reproducible across testing methods, and provide clinically meaningful information to inform treatment strategies. In reality this is not the case, with AMR surveillance activities for STIs typically weak or non-existent in many parts of the world. Molecular methods have the potential to enhance AMR surveillance, particularly for organisms that cannot easily or readily be characterised phenotypically; which is the case for most STIs. The challenges for molecular surveillance are however many and include factors such as; the mechanisms of resistance may be many or otherwise unknown, they may miss novel mutations, the technology can be expensive, they need specialised laboratories and trained staff, and that their specificity can be undermined where target sequences are shared across different species. Despite these challenges, such methods are being developed and are now finding their way into routine settings. Advances in molecular technology and expanding knowledge of resistance mechanisms continue to pave new directions in this important area.

\section{SO9.2 NEISSERIA GONORRHOEAE: ARE WE EXERTING THE SELECTIVE PRESSURE?}

doi:10.1136/sextrans-2013-051184.0048

\section{A Ison. Public Health UK, London, UK}

Treatment of gonorrhoea has historically been delivered by a single dose of a highly effective antimicrobial agent, to which resistance is not documented, to aid compliance and break transmission. Resistance in Neisseria gonorrhoeae compromises this approach and occurs both by acquisition of plasmids or chromosomal DNA from other bacteria or Neisseria spp or by selection of mutants resulting from misuse or overuse of antimicrobial agents, such as long term use of a single agent.

Resistance to ciprofloxacin, a fluoroquinolone, illustrates the effect of both misuse of earlier generations of quinolones and of suboptimal doses, as well as overuse. Quinolones target the DNA gyrase and topoisomerase enzymes that are responsible for DNA supercoiling and any interference with this process is bactericidal. Ciprofloxacin was widely used, often at low doses because of its high efficacy, but resistance emerged quickly resulting from selection of mutants, altering the target site and giving increasing drifts to resistance. Azithromycin, a macrolide which binds to $23 \mathrm{~S}$ rRNA component of the $50 \mathrm{~S}$ ribosome and interferes with protein synthesis, is effective against multiple STIs and therefore the selective pressure for resistance has been considerable. Although low-level resistance emerged quickly, high-level resistance in $N$. gonorrhoeae, resulting from a single point mutation in the peptidyltransferase loop of domain $\mathrm{V}$ of the $23 \mathrm{~S}$ rRNA gene, was only reported in recent years and threatens to compromise its use. Sporadic use of spectinomycin selected for high-level resistance in a single step, which appears clonal and has not spread widely. Limited use of the aminoglycoside, gentamicin, for which the efficacy data is weak, appears to remain clinically active.

In this era of multi-drug resistant gonorrhoea it is imperative that the selective pressure exerted by continual use of a single agent is understood and clinical practise modified, where necessary, to prevent gonorrhoea becoming untreatable.

\section{S09.3 HERPES SIMPLEX VIRUS}

doi:10.1136/sextrans-2013-051184.0049

1,2E Frobert, ${ }^{3} \mathrm{~S}$ Ducastelle-Lepretre, ${ }^{1,2} \mathrm{~J}$ Casalegno, ${ }^{1} \mathrm{~V}$ Nave, ${ }^{4,1} \mathrm{D}$ Eibach, ${ }^{1,2} \mathrm{~B}$ Lina, 1,2F Morfin. 'Laboratory of Virology, Centre de Biologie et Pathologie Est, Hospices
Civils de Lyon, Lyon, France; 'Virologie et Pathologie Humaine, Université Lyon 1, EA 4610, Faculté de Médecine RTH Laënnec, Lyon, France; ${ }^{3}$ Service d'hématologie Clinique, Centre Hospitalier Lyon Sud, Lyon, France; "European Public Health Microbiology Training Programme (EUPHEM), European Centre for Disease Prevention and Control (ECDC), Stockholm, Sweden

The more and more frequent and widespread use of acyclovir (ACV), but also the increasing number of immunocompromised patients might induce an increase in HSV (Herpes Simplex Virus) resistance. HSV resistance to ACV is mainly associated with mutations in the thymidine kinase (TK) gene although mutations in the DNA polymerase can be observed. Up to now, resistance of HSV to ACV was a major concern for immunocompromised patients with a frequency between 2.5 and $10 \%$. This study aimed to reassess HSV resistance to ACV, during a ten year period, in immunocompetent and in immunocompromised patients (bone marrow transplant patients, solid organ transplant, HIV positive patients, cancer patients). From 2002 to 2011, 1538 patients positive for HSV were tested for the susceptibility of their virus to ACV (1044 immunocompetent and 494 immunocompromised). In immunocompetent patients, prevalence of resistance remains under $0.5 \%$, whatever the period studies. In immunocompromised patients, a significant increase can be observed, from 4.3\% during 2002-2006 (11/255 patients) to $13.4 \%$ during $2007-2011(32 / 239)$ ( $p=0.0002)$. This significant increase is mainly observed among bone marrow transplant patients in which the prevalence is $10 \%(5 / 52)$ during 2002 2006 and $38 \%(30 / 79)$ during 2007-2011 ( $p=0.0002)$, whereas other types of immune deficiencies do not show an increase $(1.3 \%$ versus $2.9 \%, p=0.2)$. New chemotherapy protocols (FLAMSA) and type of transplantation as blood cord transplant are part of the explanation. Genotyping of the resistant viruses (35 viruses) reveal mutations in the TK gene for $80 \%$ of them. Double population including resistant and susceptible viruses were recovered in 5 isolates $(5 / 34=14 \%)$. Rapid diagnosis of HSV resistance, but also research on alternative treatment are more than ever of interest.

\section{SO9.4 MYCOPLASMA GENITALIUM}

doi:10.1136/sextrans-2013-051184.0050

J S Jensen. Statens Serum Institut, Copenhagen, Denmark

M. genitalium infections explain $15-25 \%$ of symptomatic male NGU and causes sexually transmitted urethritis, cervicitis, and PID in women. The bacterium is extremely difficult to isolate by culture, and consequently, the knowledge about antimicrobial resistance and its underlying molecular mechanisms has been slow to accumulate.

In the few randomised trials of $M$. genitalium conducted to date, doxycycline has been compared with a $1 \mathrm{~g}$ single dose of azithromycin, and together with results from open trials, it is evident that doxycycline is inefficient in eradicating $M$. genitalium with eradication rates around $35 \%$. The eradication rate after azithromycin $1 \mathrm{~g}$ single dose has most often been significantly higher, but differs greatly between studies. Remarkably, older studies appear to have higher eradication rates than the more recent ones, and in the latest study from the US, no significant difference between doxycycline and azithromycin efficacy could be detected.

Although several mutations have been associated with increased macrolide MIC in strains selected by passage in the presence of macrolides, only mutations in the 23S rRNA gene at position 2058 and 2059 (E. coli numbering) have been detected in patients failing azithromycin treatment.

A number of rapid methods for detection of such mutations directly from clinical samples have been developed and have proved to be clinically useful in directing treatment. Pre-treatment mutations have been found in between $10-15 \%$ of contemporary samples where doxycycline is used as the primary NGU treatment and is most commonly around $40 \%$ in settings where azithromycin is the 